\title{
Sustainable Plastics Inspired by Nature
}

\author{
Plant-based plastics offer a sustainable alternative to traditional \\ petrochemical plastics. Scientists and engineers are making the shift \\ easier by fine-tuning the structure and function of these biomaterials \\ while also developing better processing techniques.
}

By Peter Halley

$\checkmark$ ince Alexander Parkes patented the first plastic in 1856, the materials have found their way into every corner of our planet-including many where they shouldn't be. Plastic waste travels from landfills to rivers to the oceans. It leaches chemicals, contaminates food chains, and accumulates in different ecosystems, from deep-sea floors to the highest alpine glaciers. And because most of that plastic is derived from fossil fuels, it is there to stay. But the ubiquitous petroleum product doesn't have to be a permanent fixture in our society. Technical advances are now making bioplastics-polymer materials derived from plant sources (biomass) - a functional yet renewable alternative to petroleum-based plastics (Fig. 1). This development could have a potentially transformative effect on the environmental and global economy.

To mitigate the impact of the 322 million tons of plastics produced worldwide in 2014-only 22 million tons of which were recycled-plastic production and consumption needs to shift from a one-way model that ends in a landfill, to a model that sustainably integrates redesign, reuse, and recycling. To reach such a goal, researchers first need to hone their understanding of the bulk properties and chemical structure of bioplastics and then to apply that knowledge to making the processing of these materials scalable and commercially viable. On top of these technical advances, communities, industries, and governments need to find ways to work together to integrate bioplastics into a sustainable future.

\section{From Plastic to Bioplastic}

Plastics are synthetic organic compounds that are malleable

\section{ARTICLE TAKEAWAYS}

Researchers are making bioplastics an important part of a sustainable plastics industry, by improving processing techniques and addressing the full production lifecycle.

Advanced understanding of polymer structure and function is leading to bioplastics with better bulk properties and lower cost.

Community, industry and government have interactive roles to play in developing a sustainable plastics economy that's good for us and the planet.

and can be molded into solid objects. Typically, they're made from organic polymers of large molecular mass, bound together through chemical processes such as condensation, polyaddition, and cross linking. Those polymers are most commonly derived from petrochemicals. Plastics may also contain other substances that prolong the materials' lifetime or malleability. Common forms of plastics include nylon (polyamides), fibers (polyesters), and plastic bottles (polyethylene and polyethylene-terephthalate).

An array of options, however, come from renewable materials. Natural rubber and cellulose paved the way for these alternatives. Since the 1970s, those materials have been examined $[1,2]$ as an option to replace petrochemical plastics and to reduce plastics waste accumulating in landfills. Generally, a bioplastic is a polymer comprised of biomass-derived monomers that, at some stage during processing, can be molded and processed into a finished product [3]. Some bioplastics, like those derived from starch, 


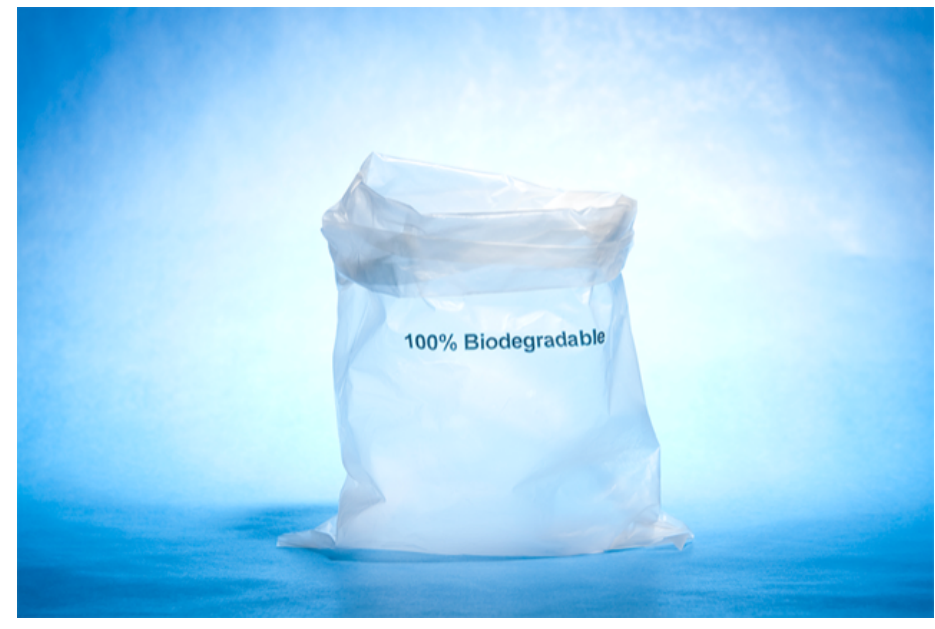

Figure 1: Petrochemical plastic waste leaches chemicals, contaminates food chains, and accumulates in ecosystems around the globe. Technical advances are now making bioplastics a functional and renewable alternative to petroleum-based plastics. Credit: timsa/E+/Getty Images

alginates, and chitosan, are biodegradable, meaning that they break down into water and carbon dioxide; others, like lignin and biomass-derived polyethylenes and polyamides, are nonbiodegradable.

For a bioplastic to be useful, its properties must be competitive with those of petroleum-based products. Such properties include resistance to moisture, ease of processing, durability, and appropriate stiffness or flexibility. Steps toward achieving such goals range from genetically engineering plants to increasing production of certain proteins and starches to replacing individual component petroleum plastics with renewable alternatives. Four main developments are poised to have great impact.

\section{Making Starch-Based Bioplastics More Durable}

Starch-based polymers are bioplastics that are produced on industrial scales and prevalent in flexible films, agricultural mulch films, food packaging, and plant pots. These bioplastics are made by blending plant-derived starches with plasticizers such as glycerol or glycol, which add malleability, and with other additives that simplify processing. They are inexpensive, biodegradable, and are made almost entirely from renewable sources. Still, they are limited by their tendency to absorb water and may degrade rapidly in environments with high temperature and humidity.

To overcome those limitations, researchers are exploring several routes. With genetic engineering, they can include carefully selected combinations of straight or branched polymers of desired molecular weights into the starches, which can lead to more durable materials [4]. With manufacturing processes that combine into a single step the synthesis of the polymer with the melting, blending, structuring, and shaping of the material, they can improve water resistance while maintaining biodegradability [4]. Finally, the addition of novel plasticizers can improve stability and decrease viscosity as well as add new functionalities or properties like electrical activity and very low gelatinization temperatures [5].

\section{Making Pliable, Economical Polyesters}

Another important group of plastics is that of polyesters, which are polymers that contain an ester functional group in their main chain and are best known for their use in fibers that are commonly made into textiles. Recent advances, made possible by new plant-based sources of polyester precursor molecules and by new techniques for synthesizing polymers from those precursors, are leading to improvements in mechanical properties, biodegradability, and economic viability. Some commercially available polyesters are renewable, but more improvements are on the horizon.

Polylactic acid, for instance, is a polyester that's already economically produced from renewable sources. Its monomer, lactic acid, is obtained by fermenting ingredients such as corn starch, sugar cane, or beet pulp. A closely related group of polyesters, polyhydroxyalkanoates, uses different monomers produced by microorganism fermentation of sugars or lipids. Both polylactic acids and polyhydroxyalkanoates are biodegradable, as they can be decomposed at high temperatures or by immersion in saline water. These plastics are good barriers to moisture and thus useful in packaging. However, they tend to be brittle and rigid. To improve the pliability of polyesters, current research focuses on techniques such as copolymerization and polymer blending, which are methods for combining different monomer components.

Another common polyester, polybutylene succinate, is a biodegradable polymer made from succinic acid monomers. 
The commercially available version, used in some packaging, is based on fossil-fuel-derived succinic acid, which is expensive. By making those monomers from renewable resources instead, studies are demonstrating possible ways to make the material more affordable [6]. The plentiful biological sources of succinic acid, such as corn starch, sugarcane molasses, whey, wood, and soybean should enable low-cost production.

More generally, research on blended copolymers (combinations of different monomer sequences in a polymer chain) has led to biodegradable polyesters with enhanced mechanical and performance properties $[7,8]$. Key steps taken in the last year include the development of copolyesters-polyesters containing two different monomers-such as poly(hydroxy-butyrate-valerate). Adjusting the monomer ratio gives control over mechanical properties such as stiffness, water permeability, and tensile strength. And the material is fully biodegradable.

\section{Making Nonbiodegradable Bioplastics More Sustainable}

"Biodegradable" has become a buzzword in the field of sustainability and is indeed a valuable property for single-use products. But for many long-term applications, a plastic that degrades rapidly is undesirable. Nonbiodegradable bioplastics can nonetheless be made sustainable, by replacing nonrenewable components with renewable ones. For example, two of the most commonly used plastics for these kinds of applications are polyethylene (PE) and polyethylene terephthalate (PET). Rather than using petrochemically derived ethylene to synthesize those polymers, researchers can produce bio-PE and bio-PET using ethylene produced from biomass-derived ethanol (Fig. 2). These materials look attractive as replacements for materials found in flexible packaging and plastic bottles, as evidenced by interest from companies like drink manufacturers Coca-Cola and Pepsi.

Other petrochemical plastics like butyl rubber, a copolymer of isobutylene and isoprene, can similarly be made in a sustainable way. Both monomer components are available from renewable biomass resources obtained by fermentation processes or by crops that are genetically engineered to optimize the production of these monomers [9]. These processes are well understood and developed; however, the specific biochemical production steps have not yet been scaled

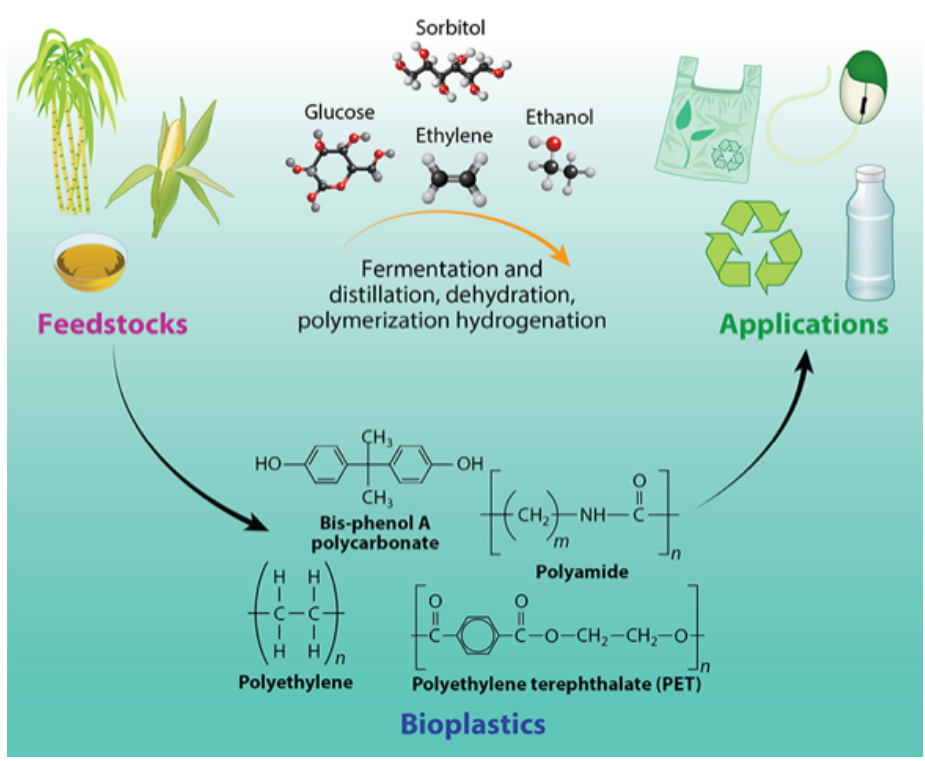

Figure 2: Nonbiodegradable bioplastics, whose component chemicals are sourced from feedstocks and synthesized via a combination of fermentation, distillation, and dehydration processes, can replace their petrochemical counterparts in many single-use applications.

Credit: APS/Carin Cain and P. Halley

up for mass production. Companies such as Genomatica are beginning to examine the possibility of scaling up biotechnology approaches that use, for example, microbial host cells to produce the needed monomers.

\section{Making High-Performance Biocomposites}

A biocomposite combines a matrix of polymers with a reinforcement of natural or nanocomposite fibers. These materials mimic the structure of living materials, while strengthening them through the matrix. The matrix can absorb mechanical load on the fibers and protect them from damage. Researchers in the bioplastics community are developing composites based on natural fibers from abaca, flax, hemp, jute, kenaf, sugar cane, and many other plants, as well as composites based on artificial nanomaterials such as layered silicates, graphene, nanotubes, and nanotubes-nanocellulose mixtures [10]. Biocomposites can be renewable, cheap, recyclable, and biodegradable. Some successful designs offer excellent mechanical properties in sheet applications, such as wood-like paneling and carpeting, useful in the construction 
and automotive industries.

Biocomposites have potential for combining the sustainability of bioplastics with the high mechanical and thermodynamic standards of traditional materials. To further improve biocomposite properties, researchers are working on improving the macroscopic interfaces between polymer and fibers to reduce voids, designing new fibrous filler materials, and improving processing techniques.

\section{Old Equipment Makes New Plastic}

The biggest metadevelopment that has boosted bioplastics recently has been the focus on materials that can be processed with existing equipment used for conventional plastic, a solution known as drop-in. Originally, many bioplastic alternatives-such as an early version of McDonald's starch-based burger packaging-had to be fabricated on specialized processing equipment. But convincing companies to invest in new equipment was a major hurdle.

Commercially available bioplastics like bio-PE and bio-PET are already produced on conventional equipment used for their petrochemical counterparts. In addition, new bioplastic polyesters and polymer blends can be designed to have melt properties close to those of conventional plastics, thus allowing for similar processing on existing equipment. When developing new bioplastics, researchers need to continue to focus on making materials compatible with existing equipment-for example, by tailoring the product's rheological characteristics via process-enhancing plasticizers and extent of polymer blending [4].

\section{Integrating Bioplastics into a Sustainable Cycle}

Building bioplastics into a sustainable economy is only partly about technical advances. To bring materials advances to fruition, researchers also must address how new materials may be sustainably integrated into the whole process, going from production to end-of-life. That means examining and optimizing the overall material and energy balance of the plastic's lifecycle (Fig. 3). For example, plastic packaging that can be composted directly with food waste may avoid energy-intensive recycling procedures; and bioplastics with low melting temperatures may require less energy to be processed [11-15].

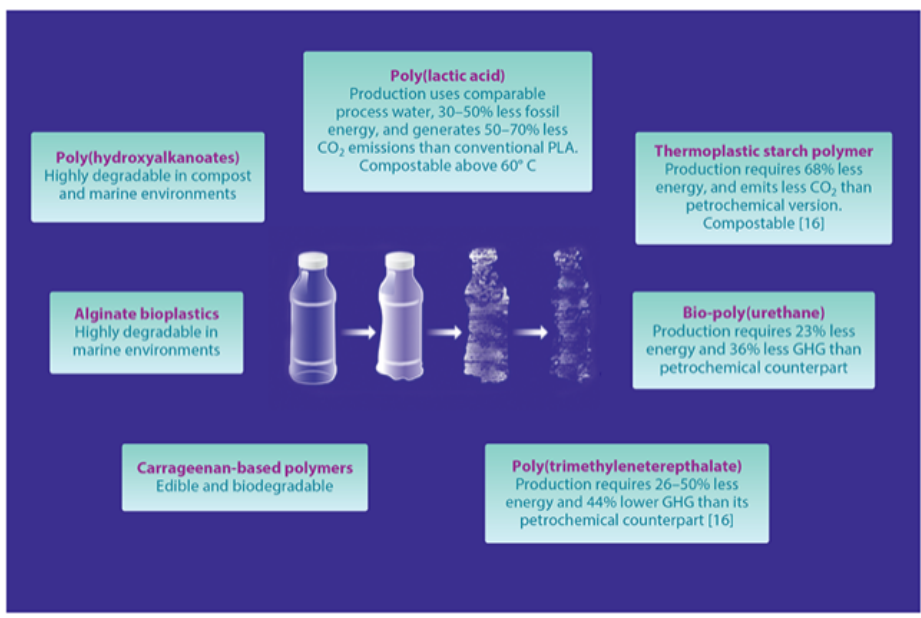

Figure 3: Biodegradable bioplastics can offer unique advantages and net sustainability improvements, noted in blue, compared to many petroleum-based ones. Those improvements are achieved at various points in the material's life cycle, from production to end-of-life (GHG = greenhouse gases).

Credit: APS/Carin Cain and P. Halley

\section{Promoting Cooperation Among Communities, Industries and Governments}

For sustainable materials to provide social and economic benefits, communities, industries, and governments must collaborate to identify solutions that can be applied at the local level. A roadmap toward a sustainable plastics economy needs to address not only technical solutions but also social and policy changes [16].

One promising example is the Plastics Pact facilitated by the Ellen MacArthur Foundation, which brings together regional and national stakeholders to find and share solutions. In one initiative, plastic producers examine how they can design products to facilitate recycling. For example, Niaga, a carpet producer, has replaced multilayer plastics with a single-layer bioplastic. In another initiative, chemical companies such as Dow develop technologies to improve the properties of products made from recycled plastics [17]. In another powerful initiative, 54 nations have formed the Alliance to End Plastic Waste, which awards grants to fund infrastructure changes including waste management and industrial composting facilities, bioplastics innovation, education and engagement, and cleanup. 
Bioplastics should be regarded as part of a holistic solution to transitioning to a sustainable plastics industry, much like biofuels are part of the greater context of a sustainable energy industry. Thus, comprehensive lifecycle assessments are necessary to find the net contribution of these materials, as their production may include energy-intensive steps. Researchers and other stakeholders all have a role to play by carefully examining energy use while sourcing biochemicals, optimizing water and energy use during bioplastic processing, and designing the product for final disposal, recycling, and collection.

Correction (22 September 2020): The subheadline was updated to refer to "scientists" instead of "physicists" because the efforts described in the article involve many scientific fields.

Peter Halley: School of Chemical Engineering, The University of Queensland, Brisbane, Australia

\section{REFERENCES}

1. R. P. Westhoff et al., "Starch-filled polyvinyl chloride plastics-preparation and evaluation," Ind. Eng. Chem. Prod. Res. Dev. 13, 123 (1974).

2. E. B. Bagley et al., U. S. Patent No. 4,026,849 (1977).

3. M. Vert, "Terminology for biorelated polymers and applications (IUPAC Recommendations 2012)," Pure Appl. Chem. 84, 377 (2012).

4. L. Avérous and P. J. Halley, Starch polymers: From genetic engineering to green applications (Elsevier, Burlington, 2014).

5. B. Zhang et al., "Facile preparation of starch-based electroconductive films with ionic liquid," ACS Sustainable Chem. Eng. 5, 5457 (2017).

6. X. Dai and Z. Qiu, "Synthesis and properties of novel biodegradable poly(butylene succinate-co-decamethylene succinate) copolyesters from renewable resources," Polym Degrad. Stabil. 134, 305 (2016).

7. M. Gigli et al., "Poly(butylene succinate)-based polyesters for biomedical applications: A review," Eur. Polym. J, 75, 431 (2016).

8. J. Jian et al., "An overview on synthesis, properties and applications of poly(butylene-adipate-co-terephthalate)-PBAT," Adv. Ind. Eng. Polym. Res. 3, 19 (2020).

9. W. Lei et al., "High performance bio-based elastomers: Energy efficient and sustainable materials for tires," J. Mater. Chem. A 4, 13058 (2016).

10. T. Gurunathan et al., "A review of the recent developments in biocomposites based on natural fibres and their application perspectives," Compos. Part A: Appl. Sci. Manuf. 77, 1 (2015).

11. Australian Packaging Covenant Organisation, Considerations for Compostable Plastic, May 2020.

12. L. S. Dilkes-Hoffman et al., "The rate of biodegradation of PHA bioplastics in the marine environment: A meta-study," Mar. Pollut. Bull. 142, 15 (2019).

13. Y. B. Farias et al., "Biodegradable sodium alginate films incorporated with norbixin salts," J. Food Process Eng. 43, e13345 (2020).

14. C. R. Álvarez-Chávez et al., "Sustainability of bio-based plastics: general comparative analysis and recommendations for improvement," J. Cleaner Prod. 23, 47 (2012).

15. F. D. S. Larotonda, "Hybrid carrageenan-based formulations for edible film preparation: Benchmarking with kappa carrageenan,” J. Appl. Polym. Sci. 133, 42263 (2015).

16. Ellen MacArthur Foundation, The New Plastics Economy: Rethinking the future of plastics (2016); Catalysing Action (2017).

17. "Milliken nucleator 'significantly increases' HDPE performance and processing," Addit. Polym. 2015, 4 (2015). 